\title{
Carbon Nanotubes in Protective Fabrics: A Short Review
}

\author{
Sushant Sharma ${ }^{1,2}$, Sanjay R Dhakate ${ }^{1}$ and Bhanu Pratap Singh ${ }^{1,2 *}$ \\ ${ }^{1}$ Advanced Carbon Products Section, India
}

${ }^{2}$ Academy of Scientific \& Innovative Research (AcSIR), India

*Corresponding author: Bhanu Pratap Singh, Advanced Carbon Products Section, CSIR-National Physical Laboratory, Dr KS Krishnan Marg, New Delhi-110012, India

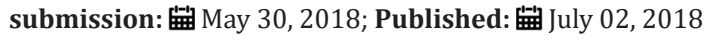

\begin{abstract}
Carbon nanotube (CNT) is a very fascinating material with outstanding properties suitable for various applications. In this review the importance of CNTs as ballistic materials are discussed, starting from various application of CNTs in textile industries and the utilization of CNTs in different ways to make composites for ballistic protection i.e dispersion of CNTs in polymer matrix which is used as impregnation of ballistic fabrics, growth of CNTs on ballistic fabrics and finally CNTs based fabrics. Some recent work carried out by various researchers in this field is also discussed. It has been found that only few layers of CNT fabrics can replace the several layers of ballistic fabrics and this will help in reducing the weight of existing ballistic protective fabrics significantly without hampering their performance.
\end{abstract}

Keywords: Carbon nanotubes;CNT fabrics; Protective fabrics; Polymer matrix; High performance fabric

\section{Introduction}

Due to the constant requirement of textile material with unique mechanical, electrical, thermal, chemical along with wearable properties, development in textile sector has come a long way. These multifunctional textiles utilized in various conventional and innovative sectors like sportswear, electronics, automobile sector, geo-textile, bio-medical textile, protective clothing for law enforcement, etc. The quest of textile research for versatile applications comes to a new horizon called "Carbon Nanotube"or CNT.

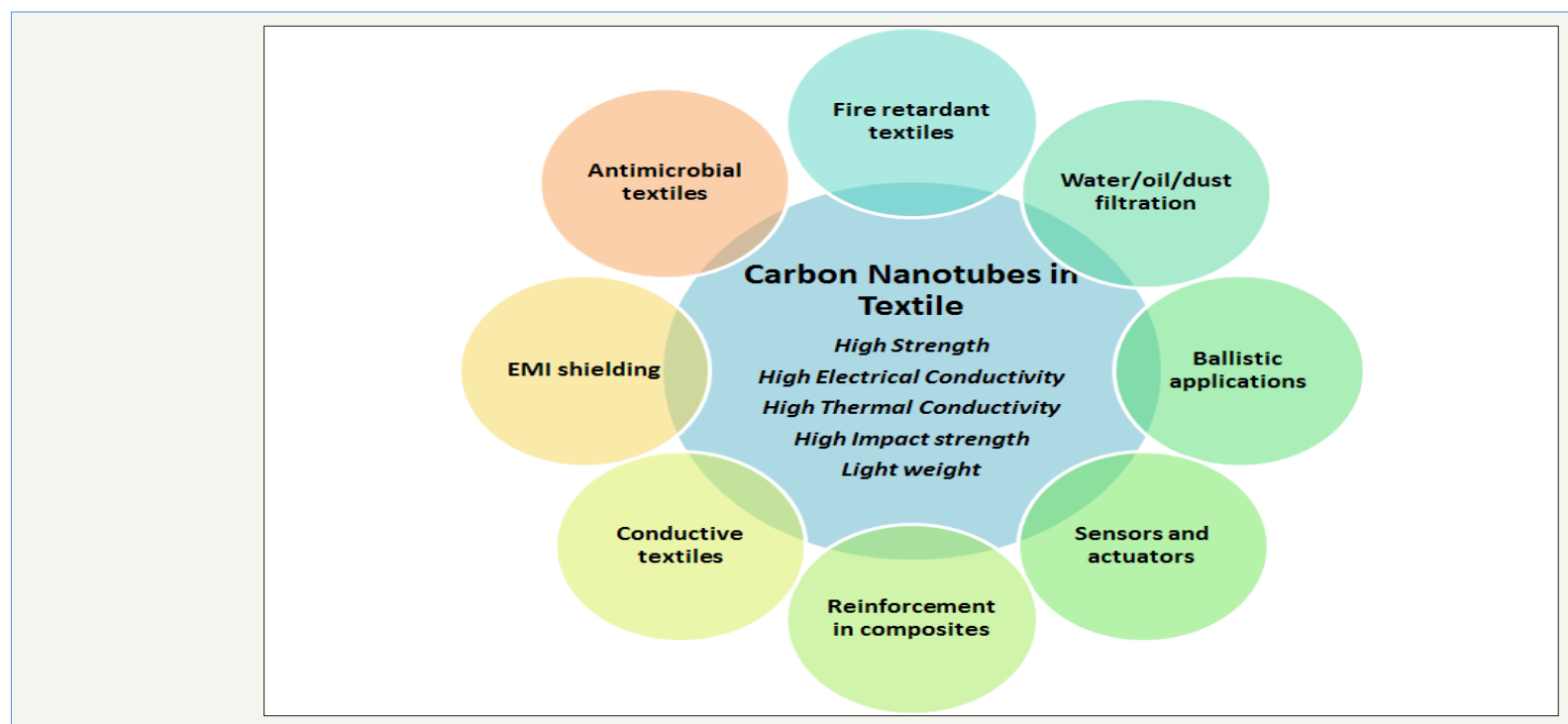

Figure 1: CNT application in textile industries.

Carbon nanotube is a one dimensional, $\mathrm{sp}^{2}$ hybridized carbon having outstanding mechanical, electrical, thermal, optical and ballistic properties and can be applicable in different industries as manufacturing, medicine, nanotechnology, electronics, sports goods, construction, polymer, and textile[1]. CNTs have various applications in textile industries as shown in Figure 1. 
CNTs possess high stiffness, high toughness, and high energy absorption capacity as compared to other well-adopted ballistic fibers. This short review provides some updated details on application of CNT as ballistic protection fabric. An earlier study by Mylvaganamet al. [2] gives the molecular dynamic simulation for analyzing CNT and its mechanical performance, which was surprisingly high and suitable for high mechanical energy storage textile application [2]. Further, Mylvaganam et al.[3] evaluated the ballistic response of CNT theoretically and experimentally $[3,4]$. The studies [2,3] involved various parameters such as projectile velocity, projectile distance, effect of clamping, and physical aspects of CNT which provides a dynamic model.This model is further carried forward by Zhang et al. [5] in macro scale in which firstly CNT forest was synthesized by chemical vapour deposition method and the fibers were prepared by spinning method. They[5] further studied the mechanical properties of these fibers experimentally and concluded that CNT fibers are stronger, stiffer and light weight than that of any other commercially available ballistic fibers. These remarkable properties of CNTs and its fiber make them an ideal candidate for employing them in different combinations with polymers, woven fabrics, fibers as well as ceramic alloys to make hybrid composites for coping with high velocity projectile threats.

CNTs can be used in three different ways to improve the performance of ballistic-resistance material. Firstly, the ballistic grade reinforced fabric along with CNT to enhance the energy absorption capacity. Secondly, CNTs grow over the surface of high performance fabric by several synthesis techniques. Thirdly, CNTs can be used in the form of woven and non-woven fabric for achieving outstanding ballistic resistance performance.

High velocity projectile resistant fabrics are generally made up of high performance spin-yarn which are woven in twodimensional (2D), three-dimensional (3D) pattern formed by interlacing in warp and weft direction orthogonally. A stress wave is generated at a point of impact when high velocity projectile strike the fabric and it travels along longitudinal and transverse direction simultaneously. The speed of stress wave in the fabric depends on the stiffness and the density of the ballistic fiber. The kinetic energy of the stress wave is dissipated through fiber deformation, inter fiber friction etc. therefore causes deformation and delamination in fabric. Hence, it was observed that friction plays an important role in improving the overall performance of the impact resistant fabrics. To overcome this problem, Hymas et al. [6]developed a new functionalization method which is first realized theoretically by simulation and afterwards experimentally. In the study[6], Kevlar 29 is modified by adding an embedded cross-linked network of poly butyl acrylate (PBA) functionalized CNT. Mechanical and ballistic test are performed which reveals that modified Kevlar with CNT network increases the projectile impact resistance with no expense of flexibility. Most recently, Sharma et al.[7]improved the mechanical properties of Kevlar 49 fabric using long length MWCNTs. It was realized that the length of MWCNT improves the in-plane and through-plane mechanical properties by bridging action. The addition of longer length of MWCNTs improves the load carrying capacity of Kevlar fabric by improving its interfacial properties.

CNTs are the unique tool in nanotechnology owing to it exceptional properties, but their low dispersibility in polymer and aqueous media hampers their development in textile industries. Therefore, researchers are working towards various surface growth methods to enhance the direct uniform growth of CNTs over fiber surface. Bajpai et al. [8]developed an extremely fast growth method to synthesize CNTs over carbon fiber by using microwave irradiation method [8]. In this experimental study, a mixture of graphite, ferrocene, and carbon fiber is irradiated by microwave. It is observed that within just $5 \mathrm{sec} \sim 26 \mathrm{wt}$. \% of CNTs are synthesized which is a very fast and high yield technique for growing CNTs uniformly over the surface of fiber and it can be useful in large scale production of CNTs integrated carbon fiber. With this ideology, Hazarika et al. [9] synthesized the free standing CNTs on Kevlar fiber surface and further reinforced it to prepare polyester composite. Kevlar fibers were initially decorated with polypyrrole (PPy) and irradiated with microwave to synthesis nano projections of CNTs over it followed by vacuum bag impregnated with Ag-graphene dispersed in polyester resin. It was observed that, the synergistic of CNTs and graphene is helpful in improving the mechanical and impact resistance properties by high polymer interlocking mechanism [9].

Synthesizing CNTs over fabric surface is a useful technique to control the projectile resistance performance of the fabric by maintaining the dispersibility, uniformity and strong covalent interaction between fabric and nano-projection. But, still the properties of CNTs are not fully realized in this regard. Alignment and loading of CNTs are the major factors which are necessary for governing the further improvement in CNTs based fabric. Today, there are several techniques such as wet spinning, forest spinning and aerosol spinning etc. commonly employed for producing high performance macroscopic CNTs yarns and their fibers[10,11]. These CNT fibers have tremendous mechanical and electrical properties which can be used in wearable smart textile. Delton et al. [12] synthesized the super tough CNTs composite fiber of 100 $\mathrm{m}$ long by using coagulation based technique and weaved it in 2D fashion to produce the CNT fabrics [12].

\section{Conclusion}

CNTs are the futuristic material for protective clothing due to its light weight, high strength and high energy absorption capacity and have created an interesting field in textile industry for continuous investigation. For fabricating light weight multi-purpose protective fabric, CNT is a promising candidate. It will not only replace the several layers of ballistic fabrics by single layer T-shirt but can also be used as fire inhibiter and UV protection textile as well.

\section{References}

1. Shahidi S, Moazzenchi B (2018) Carbon nanotube and its applications in textile industry-A review. The Journal of The Textile Institute. 2018: 
$1-14$.

2. Mylvaganam K, Zhang L (2004) Important issues in a molecular dynamics simulation for characterising the mechanical properties of carbon nanotubes. Carbon 42(10): 2025-2032.

3. Mylvaganam K, Zhang L (2006) Energy absorption capacity of carbon nanotubes under ballistic impact. Applied Physics Letters 89(12): 123127.

4. Mylvaganam K, Zhang L (2007) Ballistic resistance capacity of carbon nanotubes. Nanotechnology 18(47): 475-701.

5. Zhang X, Li Q, Holesinger TG, Arendt PN, Huang J, et al. (2007) Ultrastrong, stiff, and lightweight carbon-nanotube fibers. Advanced Materials 19(23): 4198-4201.

6. Hymas C, Lacey S, Rohrbach K, Gillard S, Berkey C. Novel PBA-grafted carbon nanotube soft body armor. pp. 1-24.

7. Sharma S, Pathak AK, Singh VN, Teotia S, Dhakate S, et al. (2018) Excellent mechanical properties of long length multiwalled carbon nanotube bridged Kevlar fabric. Carbon 137: 104-117.

8. Bajpai R, Wagner HD (2015) Fast growth of carbon nanotubes using a microwave oven. Carbon 82: 327-336.

9. Hazarika A, Deka BK, Kim D, Kong K, Park Y-B, Park HW (2017) Microwave-synthesized freestanding iron-carbon nanotubes on polyester composites of woven Kevlar fibre and silver nanoparticledecorated graphene. Scientific reports 7: 403-486.

10. Liu K, Sun Y, Zhou R, Zhu H, Wang J, Liu L, et al. (2009) Carbon nanotube yarns with high tensile strength made by a twisting and shrinking method. Nanotechnology 21(4): 045708.

11. Zhu H, Xu C, Wu D, Wei B, Vajtai R, et al. (2002) Direct synthesis of long single-walled carbon nanotube strands. Science 296(5569): 884-886.

12. Dalton AB, Collins S, Munoz E, Razal JM, Ebron VH, et al. (2003) Supertough carbon-nanotube fibres. Nature 423(6941): 703.
Creative Commons Attribution 4.0 International License

For possible submissions Click Here
Submit Article

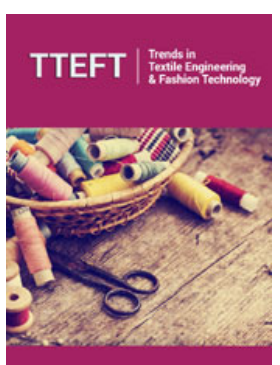

Trends in Textile Engineering \& Fashion Technology

\section{Benefits of Publishing with us}

- High-level peer review and editorial services

- Freely accessible online immediately upon publication

- Authors retain the copyright to their work

- Licensing it under a Creative Commons license

- Visibility through different online platforms 